\title{
Theoretical study of the ultrashort dynamics in graphene after fs-laser excitation
}

\author{
Sergej Krylow ${ }^{*}$, and Martin E. Garcia \\ Theoretical Physics II, University of Kassel, 34132 Kassel, Germany
}

\begin{abstract}
We report on the calculation of the full dynamics of graphene after ultrafast laser excitation using ab-initio molecular dynamics simulations. Our calculations show a biexponential decay of the (100) and (110) Bragg peaks in time, similar to experimental results. Furthermore, we calculate the time-evolution of the phonon energies averaged over sets of phonons, chosen by their phonon frequencies. Our results suggest that the fast decay time can be accounted by phonon-phonon interactions rather than the generation of SCOPs. The slower decay time is governed by the equilibration of the system with low frequency phonon modes.
\end{abstract}

\section{Introduction}

Graphene and graphite exhibit a strong coupling between the electronic system and a few optical phonons, so called SCOPs, at the Gamma and $\mathrm{K}\left(\mathrm{K}^{\prime}\right)$ point [1]. The strong coupling can be seen, for example, in the non-analytical behaviour of the SCOPs within the phonon dispersion [2] and in the signal of pump-probe experiments $[3,4]$. In the latter case, one can observe a biexponential decay of the signal. The short decay time is usually accounted to the coupling of the SCOPs with the electronic system, and the long decay time to the coupling of the SCOPs with the other phonon modes. Up to now, theoretical studies focus either on the electron-phonon coupling [2] or on the phonon-phonon interactions between SCOPs and the other phonon modes [5]. In our work we focus on the full dynamics of laser excited graphene based on ab-initio molecular dynamics simulations.

\section{Simulation Details}

For the calculations of the dynamics in laser-excited graphene we use our in-house code CHIVES, which is an ab-initio molecular dynamics code [6]. For the calculations we use periodic boundary conditions, a k-grid of $2 \times 2 \times 1$ and a supercell of graphene with 180 atoms. To simulate a monolayer we set the lattice parameter in z-direction so that no interactions between layers of graphene are possible. In order to account for the thermal motion we thermalize the system to an approximate temperature of $300 \mathrm{~K}$. After the thermalization we generate 20 different sets of positions and velocities in order to perform averages of the analyzed quantities. The coupling between electrons and SCOPs is modelled by applying an electronic temperature corresponding to an incident fluence of about $5 \mathrm{~mJ} / \mathrm{cm}^{2}$. The electronic temperature is set to decay exponentially with a decay time of $90 \mathrm{fs}$ and the approximate energy decrease of the electronic system is transferred to the SCOPs. Note that in our approach SCOPs and electrons are not coupled explicitly.

*Corresponding author: krylow@physik.uni-kassel.de 


\section{Results}

After computing the time-evolution of the system we calculate the time-dependent peak intensities for the (100) and (110) peaks, respectively. The normalized peak intensities are given by

$$
\frac{I(\{\boldsymbol{q}\}, t)}{I(\{\boldsymbol{q}\}, t=0)}=\frac{\sum_{j}\left(\frac{\left|\sum_{i=1}^{N} \exp \left(i \boldsymbol{q}_{j} \cdot \boldsymbol{r}_{i}(t)\right)\right|^{2}}{N}\right)}{I(\{\boldsymbol{q}\}, t=0)},
$$

where we sum over all wavevectors $\boldsymbol{q}$ of the particular family of peaks and over all atoms $i$ in the unit cell. The intensity quotient is shown in Fig. 1.

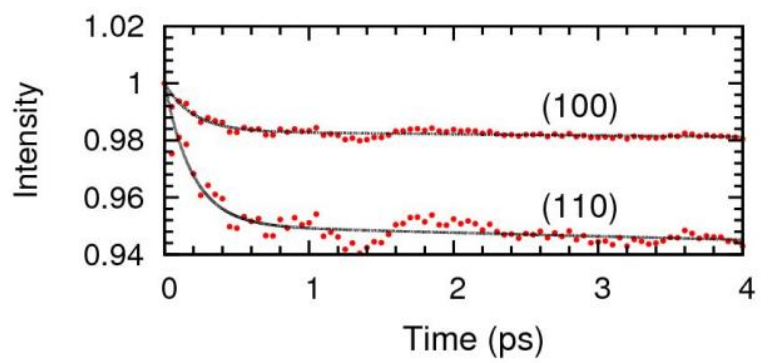

Fig. 1. Time-evolution of the (100) and (110) intensity peaks in graphene after ultrafast laser excitation. Red dots refer to the ab-initio simulations and the black lines to the fitted curves.

One can see that the intensities follow a biexponential decay, which was previously seen experimentally for graphene and graphite [3, 4]. By fitting a biexponential decaying function to the data one can obtain both decay times. The short decay time is $200 \mathrm{fs}$ and is slower than the SCOP generation, which is set to $90 \mathrm{fs}$. Thus, the fast drop of the signal cannot be due to the SCOP generation but has its origin in the phonon-phonon interaction. The long decay time is 36 ps and is substantially longer than previous findings, which do not exceed 12 ps [3]. However, the error for the long decay time is large because we only fit the first 4 ps. By increasing the simulated time period, the fitted decay time for the slow decay would probably decrease. Another reason for the difference between our calculations and experiments is that we do not have defects. Generally, one can expect that defects cause a faster thermalization of the system.

Based on our calculations, we are able to obtain the averaged energies in each phonon mode. Furthermore, we perform averages over sets of phonon modes chosen by their phonon frequencies. The 108 modes with the lowest frequency constitute the first set, the next 108 phonons the second set and so on. Note, that the SCOPs and the acoustic phonons at $\Gamma$ are excluded from these averages. Figure 2 shows the averaged phonon energy for the defined sets. The inset indicates the frequency range in which the phonons of the set are included. One can clearly see that in the first $200 \mathrm{fs}$ the average phonon energy of all phonon modes is increasing similarly, probably leading to the fast decay. After this first dynamics mostly high-frequency phonon modes are generated. Then, on a slower timescale mid frequency phonons are generated and, even slower, low frequency phonon modes. One can clearly see that the bottleneck for the thermalization of the system are the low frequency phonon modes, which couple weakly to the other modes. Furthermore, by analysing the time-evolutions of the single phonon energies we see that the strong increase in the mid frequency phonon modes after about $400 \mathrm{fs}$ is caused by just 10 phonon modes out of 216. Thus, also the remaining mid frequency phonon modes thermalize slowly. That the high frequency phonon modes couple stronger to the SCOPs in the beginning indicates 
a quadratic dependence of the scattering onto the phonon occupation of the SCOPs. If the phonon occupation of the SCOPs decreases sufficiently the decay path to the mid frequency phonon modes becomes dominant. This is consistent with our recent findings for antimony in Ref. [7].

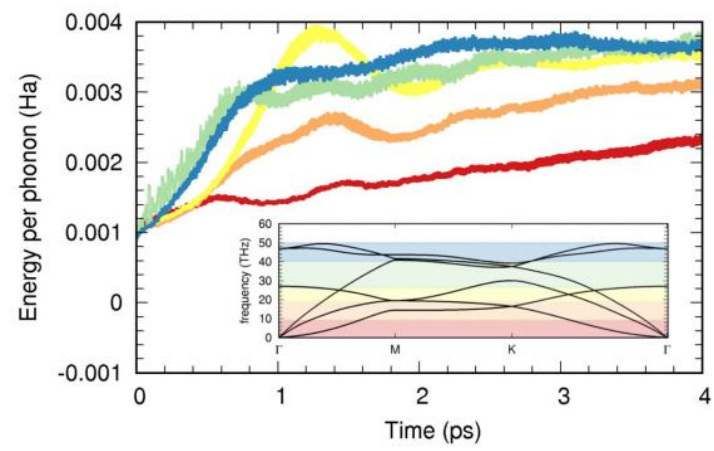

Fig. 2. The time-evolution of the Phonon energies averaged over 20 runs and sets of phonon modes (see description) after ultrafast laser excitation in graphene. The inset shows the phonon dispersion of graphene, where the used sets of phonon modes are indicated by the colours.

\section{Conclusions}

In conclusion, we have shown that our approach shows a biexponential decay of the (100) and (110) intensity peaks of graphene after ultrafast laser excitation. By calculating the energies within the phonon modes, we were able to see the detailed dynamics of the phonon modes. We see that the fast decay time is caused by phonon-phonon interactions within the first 200 fs. The slower decay time corresponds to the thermalization of the system. In detail we see that firstly high frequency phonon modes thermalize, then a few mid frequency phonon modes and finally the remaining phonon modes. In the next step of our calculations we will implement a two temperature model for the electron-phonon coupling and repeat the calculations.

\section{References}

1. J. Maultzsch, S. Reich, C. Thomsen, H. Requardt, and P. Ordej ón, Phys. Rev. Lett. 92, $075501(2004)$

2. S. Piscanec, M. Lazzeri, F. Mauri, A. C. Ferrari, and J. Robertson, Phys. Rev. Lett. 93, 185503 (2004)

3. Schäfer, W. Liang, and A. H. Zewail, New Journal of Physics 13, 063030 (2011)

4. R. P. Chatelain, V. R. Morrison, B. L. M. Klarenaar, and B. J. Siwick, Phys. Rev. Lett. $113,235502(2014)$

5. N. Bonini, M. Lazzeri, N. Marzari, and F. Mauri, Phys. Rev. Lett. 99, 176802 (2007)

6. E. S. Zijlstra, T. Zier, B. Bauerhenne, S. Krylow, P. M. Geiger, M. E. Garcia, Appl. Phys. A, 114, Issue 1, pp 1-9 (Jan 2014)

7. S. Krylow, E. S. Zijlstra, F. C. Kabeer, T. Zier, B. Bauerhenne, M. E. Garcia, Phys. Rev. Mat. 1, 073601 (2017) 